\title{
Surface Analysis of Native Spider Draglines by FE-SEM and XPS
}

\author{
Hiromitsu Sogawa ${ }^{1 \dagger}$, Kyohei Nakano ${ }^{2 \dagger}$, Ayaka Tateishi ${ }^{1}$, Keisuke Tajima ${ }^{2}$ and \\ Keiji Numata ${ }^{1 *}$ \\ ${ }^{1}$ Biomacromolecules Research Team, RIKEN Center for Sustainable Resource Science, Saitama, Japan, ${ }^{2}$ Emergent \\ Functional Polymers Research Team, RIKEN Center for Emergent Matter Science, Saitama, Japan
}

\section{OPEN ACCESS}

Edited by:

Nicola Maria Pugno,

University of Trento, Italy

Reviewed by:

Joanna Mystkowska,

Białystok Technical University, Poland

Gregory P. Holland,

San Diego State University,

United States

*Correspondence:

Keiji Numata

keiji.numata@riken.jp

${ }^{t}$ These authors have contributed

equally to this work

Specialty section:

This article was submitted to

Biomaterials,

a section of the journal

Frontiers in Bioengineering and

Biotechnology

Received: 14 September 2019

Accepted: 05 March 2020

Published: 20 March 2020

Citation:

Sogawa H, Nakano K, Tateishi A,

Tajima K and Numata K (2020)

Surface Analysis of Native Spider

Draglines by FE-SEM and XPS.

Front. Bioeng. Biotechnol. 8:231.

doi: 10.3389/fbioe.2020.00231
Although the physical and biological functions of the skin layer of spider dragline have been studied and partially clarified, the morphology and elemental contents of the skin layer of silk fibers have not been investigated in detail to date. Here, the surface of Nephila clavata spider dragline was evaluated by field emission scanning electron microscopy (FE-SEM) and X-ray photoelectron spectroscopy (XPS) to obtain clear surface morphological and molecular information. The FE-SEM images of the spider dragline indicate that the spider dragline forms a bundle of microfibrils. This hierarchical structure might induce faint fibrilar and network-like patterns on the surface of the dragline. XPS analysis revealed the presence of $\mathrm{Na}, \mathrm{P}$, and $\mathrm{S}$, which are reasonably explained by considering the biological components of the major ampullate gland of spiders. The results obtained here are preliminary but will be important to consider the molecular transition of silk proteins to form excellent hierarchical structures during the spider dragline spinning process.

Keywords: spider dragline, silkworm, silk fiber, FE-SEM, XPS, surface analysis

\section{INTRODUCTION}

Silk fibers are well known as tough biomaterials, especially spider dragline silk, which possesses excellent toughness due to a combination of great tensile strength and ductility (Blackledge, 2012; Holland et al., 2018). The physical properties of dragline silk fibers depend not solely on protein constituents but also on the hierarchical microstructure (Sponner et al., 2007; Heim et al., 2010). Previously, it was hypothesized that the hierarchical structure of spider silk fiber is similar to that of silkworm silk fiber (Gosline et al., 1986; Vollrath, 1992). However, a highly organized skin-core structure of spider silk fiber was observed by atomic force microscopy (Li S.F. et al., 1994; Li S.F.Y. et al., 1994; Brown et al., 2012; Faugas et al., 2013) and light and electron microscopy (Thiel et al., 1994; Vollrath et al., 1996; Frische et al., 1998; Shao et al., 1999; Augsten et al., 2000), and this structure was different from the proposed structure of silkworm silk fiber (Gosline et al., 1986; Vollrath, 1992). Supercontracted spider silk fiber and the phenomenon of "supercontraction" were also used to investigate a structural hypothesis consisting of a fibril core surrounding an outer thin layer (Vollrath et al., 1996; Faugas et al., 2013). An experimental procedure to separate the different skin layers of dragline fibers has been developed (Sponner et al., 2007; Yazawa et al., 2019), and the results indicate that the order of the multilayer organization from exterior to interior is as follows: lipid coat, glycoprotein, skin, outer core, and inner core (Sponner et al., 2007). Additionally, Riekel et al. reported that the surface of the spider dragline consists of highly oriented protein chains 
forming an approximately $100 \mathrm{~nm}$ skin layer based on scanning transmission X-ray diffraction measurements (Rousseau et al., 2007; Riekel et al., 2017).

The skin layer is also important for the gas barrier properties of silk fibers. The mechanical and physical properties of spider dragline and silkworm silk fibers are known to depend on the humidity; namely, the amorphous and crystalline regions are influenced by water molecules (Fu et al., 2009; Yazawa et al., 2016; Malay et al., 2017). Spider dragline is a composite of multiple biopolymers that consists of a protein core surrounded by outer skin layers. Recently, we investigated the skin layer of Nephila spider dragline, especially the biological and physical functions of the skin layers (Yazawa et al., 2019). The mechanical/physical properties as well as the crystal structures do not depend significantly on the skin constituents, suggesting that the protein core region of spider dragline determines the structural and mechanical properties. Surprisingly, the skin layer does not influence supercontraction, i.e., the layer does not have water vapor barrier properties, but does protect the fiber from protease degradation activity. This result implies that the skin layer is more important as a biological barrier than a physical barrier.

Although the physical and biological functions of the skin layer of spider dragline have been studied and partially clarified, the morphological and elemental characterizations of the skin layer of silk fibers have not been investigated in detail to date. Here, the surface of the Nephila clavata spider dragline was evaluated by field emission scanning electron microscopy (FESEM) and X-ray photoelectron spectroscopy (XPS) to obtain clear surface morphological and molecular information.

\section{EXPERIMENTAL}

\section{Collection and Preparation of Spider Dragline}

Spider draglines (silk fibers) were collected from Nephila clavata females (body sizes: approximately $25 \mathrm{~mm}$ ). Draglines were reeled at $21 \mathrm{~mm} / \mathrm{s}$ and kept in lightproof boxes at a relative humidity from 30 to $50 \%$ to prevent UV damage and drying of the silk fibers.

\section{Preparation of Spider Silk Fibers Without a Skin Layer}

The removal of the skin layer was conducted according to a previously reported method (Sponner et al., 2007; Yazawa et al., 2019). Briefly, N. clavata spider dragline silk fibers were immersed twice in diethyl ether for $10 \mathrm{~min}$. To remove the diethyl ether, the fibers were dried overnight in a vacuum oven at $40^{\circ} \mathrm{C}$.

\section{Field Emission Scanning Electron Microscopy}

The surface morphology of the spider dragline was observed and characterized by field emission scanning electron microscopy (FE-SEM, GeminiSEM, Carl Zeiss, Oberkochen, Germany). The silk fiber samples were mounted on an aluminum stub with conductive tape without sputter-coating prior to FE-SEM visualization. For the observation, the acceleration voltage and working distance were set at $0.5-1.0 \mathrm{kV}$ and $2.0 \mathrm{~mm}$, respectively.

\section{Enzymatic Treatment}

For the enzymatic treatment, we used the native draglines without the diethyl ether wash. The native draglines were immersed in $0.1 \mathrm{M}$ phosphate buffer $(\mathrm{pH}=7.4)$ containing $0.5 \mathrm{mg} / \mathrm{mL}$ of proteinase $\mathrm{K}$. Less than $1 \mathrm{mg}$ of spider dragline was immersed in $1 \mathrm{~mL}$ of the enzyme solution at $37^{\circ} \mathrm{C}$ for $24 \mathrm{~h}$. The enzymatic degradation conditions were determined based on previous reports on enzymatic degradation (Li et al., 2003; Numata et al., 2005, 2008, 2010; Numata and Kaplan, 2010). After the enzymatic treatment, the sample was washed with 0.1 $\mathrm{M}$ phosphate buffer and Milli-Q water twice and dried for 1 day under vacuum conditions.

\section{XPS Measurements}

X-ray photoelectron spectroscopy (XPS) was performed with a photoelectron spectroscopy system (PHI 5000 Versa Probe II, ULVAC-PHI). Monochromated Al K $\alpha(1486.6 \mathrm{eV})$ radiation with an operating power of $50 \mathrm{~W}(15 \mathrm{kV}$ voltage) was used in all the XPS measurements. The analyzed area was $200 \mu \mathrm{m}$. The XPS survey spectra (Figure 3B) were measured with a pass energy of 117.4 and $0.125 \mathrm{eV}$ energy step. For the measurement of each atomic element, pass energy of 23.5 and $0.025 \mathrm{eV}$ energy steps were used. The recorded signals were accumulated eight times for $\mathrm{C} 1 \mathrm{~s}, \mathrm{O} 1 \mathrm{~s}$, and twelve times for other elements. A take-off angle was $45^{\circ}$ to the sample substrate. During the measurement, the samples were neutralized using both a low-energy ion beam and a low-energy electron beam.

For data analysis, we used PHI MultiPak software (ULVACPHI). The signal background of each component was subtracted using the Shirley method. The atomic concentration was calculated by considering the relative sensitivity factor for each element corrected with the sensitivity factor of the system. The

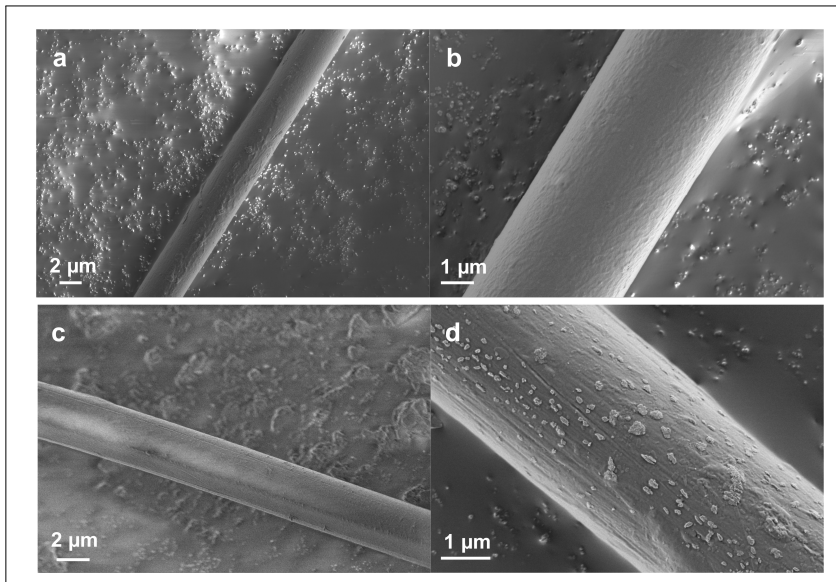

FIGURE 1 | FE-SEM images showing the surface of silk fibers at different magnifications. (a,b) Native Nephila spider dragline. (c,d) Nephila spider dragline after ether extraction. 


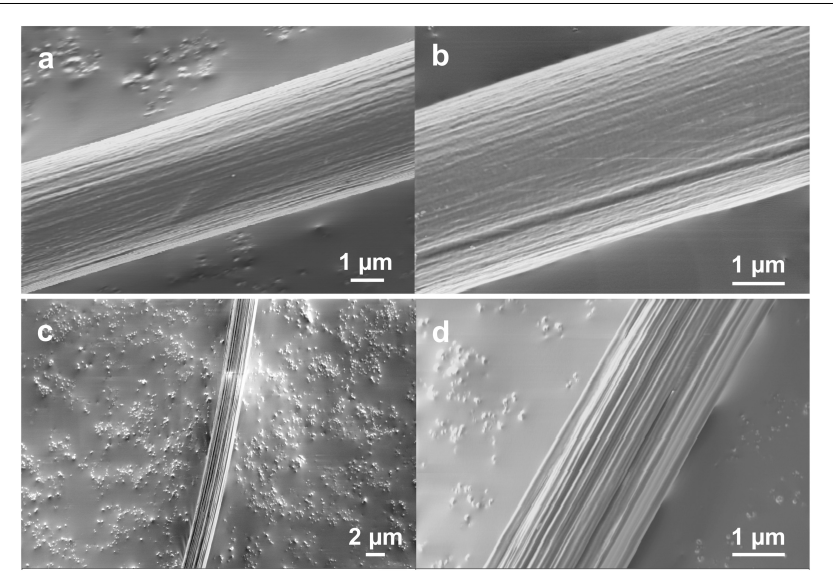

FIGURE 2 | FE-SEM images showing the surface of Nephila spider dragline after proteinase-K treatment. $(\mathbf{b}, \mathbf{d})$ Are enlarged images of $(\mathbf{a}, \mathbf{c})$, respectively.

fitting protocol used the Gaussian-Lorentzian function, but the best fit results were obtained with Gaussian 100\%.

\section{RESULTS AND DISCUSSION}

\section{FE-SEM Observations of Silk Fibers}

FE-SEM observations have several advantages over general SEM imaging. In this study, direct imaging of a single dragline without a sputter-coating process was conducted to observe the surface morphology at high resolution. Figure 1 shows the typical FESEM images of the native Nephila dragline and the dragline without a skin layer. In previous literature with SEM imaging (Lin et al., 2017; Yazawa et al., 2019), the surface of spider draglines has been reported to be smooth and clean. However, we found with FE-SEM that the surface of the Nephila spider dragline has fibril and network-like patterns (Figure 1b). Once the silk layer was washed out with ether, the patterns disappeared, but debris from the skin layer was observed at the fiber surface (Figures 1c,d). The white spots indicate the residues of the lipid layer, which is the outermost layer of dragline silk. As a comparison, the surface

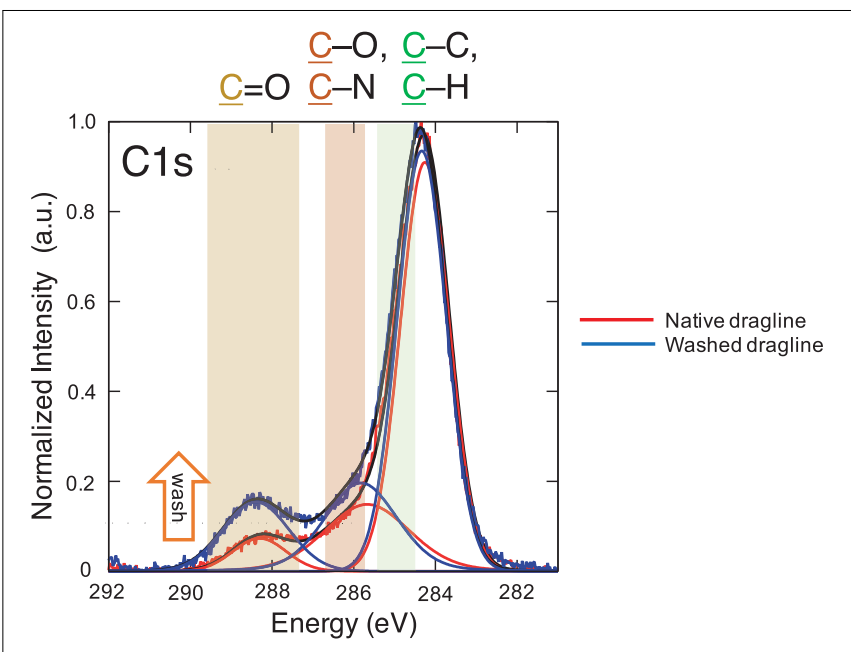

FIGURE 4 | XPS spectra of the native spider dragline (red) and spider dragline after washing with ether (blue). The peak fitting results are also shown in the same color. The details on the peak fitting is described in Experimental section.

morphologies of silkworm silk, which were previously reported and summarized, were referenced (Malay et al., 2016). In many cases, inorganic compounds have been observed on the surface of silkworm cocoon silk fibers (Numata et al., 2015). These previous reports on the surface morphologies of silkworm silks differ from the patterns found on the surface of the spider dragline. In this study, the silk fibers were not pretreated for FE-SEM imaging, and hence, the surface morphologies should not be induced by the sample treatment. Therefore, the natural spider dragline seems to show characteristic fibril and network-like patterns. The patterns might be related to the spinning process of spiders, especially the final part around the spinneret.

\section{Enzymatic Treatment}

To further examine the surface morphologies, especially the fibril and network-like patterns, we studied the morphological changes of the native Nephila spider dragline after enzymatic treatment with proteinase $\mathrm{K}$. We treated the spider dragline with
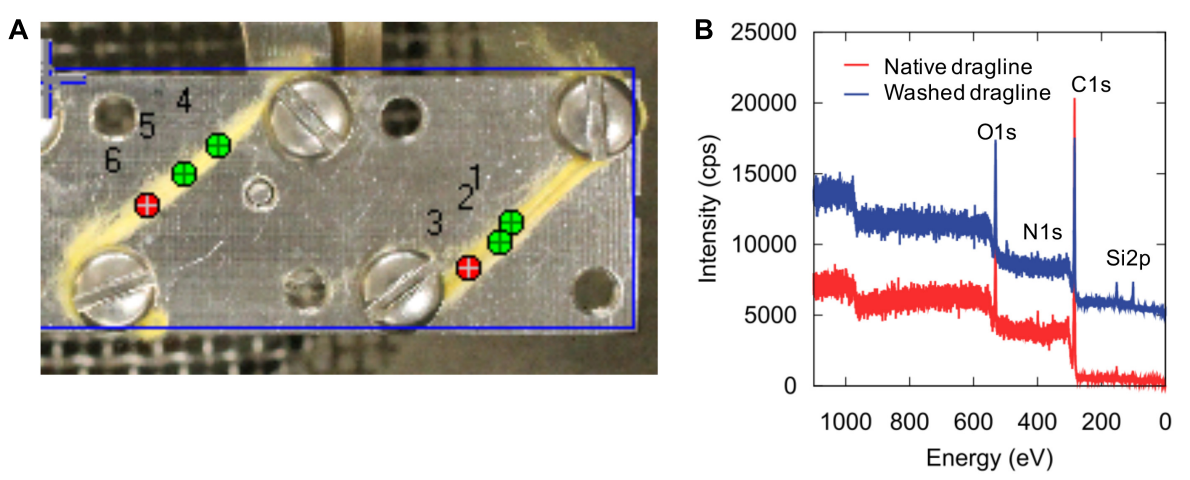

FIGURE 3 | XPS sample setting (A) and wide-scan spectra (B) of native spider dragline (red) and spider dragline after ether wash (blue). 
proteinase K (without any diethyl ether treatment), an efficient protease, and subsequently observed the dragline by FE-SEM (Figure 2). To avoid misunderstanding of the biodegradability of the spider dragline, we need to clear the biodegradability of spider dragline, according to the previous study (Yazawa et al., 2019). Yazawa et al. (2019) reported that the skin layer of the dragline can protect the dragline from the enzymatic attacks. Proteinase $\mathrm{K}$, which was found in extracts of the fungus Engyodontium album, is a serine protease with a broad substrate specificity and high activity against aliphatic and aromatic amino acids. The biodegradation of the spider dragline by proteinase $\mathrm{K}$ is not expected in the natural environments, because Nephila spider does not prefer to construct web and territories in the environments contaminated with fungi.

The enzymatic degradation behavior of the dragline was not consistent, as Figures 2a,c show different degradation degrees of the draglines. Figures $\mathbf{2 a} \mathbf{a} \mathbf{b}$ clearly shows that the dragline consists of a bundle of microfibrils. Furthermore, Figures 2c,d indicates that the bundle structures of microfibrils are maintained even inside of the dragline. Thus, both FE-SEM images indicate that the spider dragline forms a structure similar to a bundle of microfibrils. The enzymatic treatment degraded the skin layer and silk fibroins of the native draglines, resulting that these microfibrils were exposed and observed by FE-SEM. This hierarchical structure might induce faint fibril patterns at the surface of the dragline, however, we need to further study the effect of the natural spinning process with spider spinnerets.

\section{XPS Measurements}

XPS is a powerful method for characterizing the surface chemical composition and chemical states of each element. An XPS study of recombinant silk protein during a thermal degradation process was reported previously (Dao et al., 2017), but natural spider draglines have not been investigated to date. Figure 3 shows the setting of the fiber samples and the wide-range XPS spectra of the native dragline and the dragline washed with diethyl ether for skin layer removal. The peaks originating from silicon were
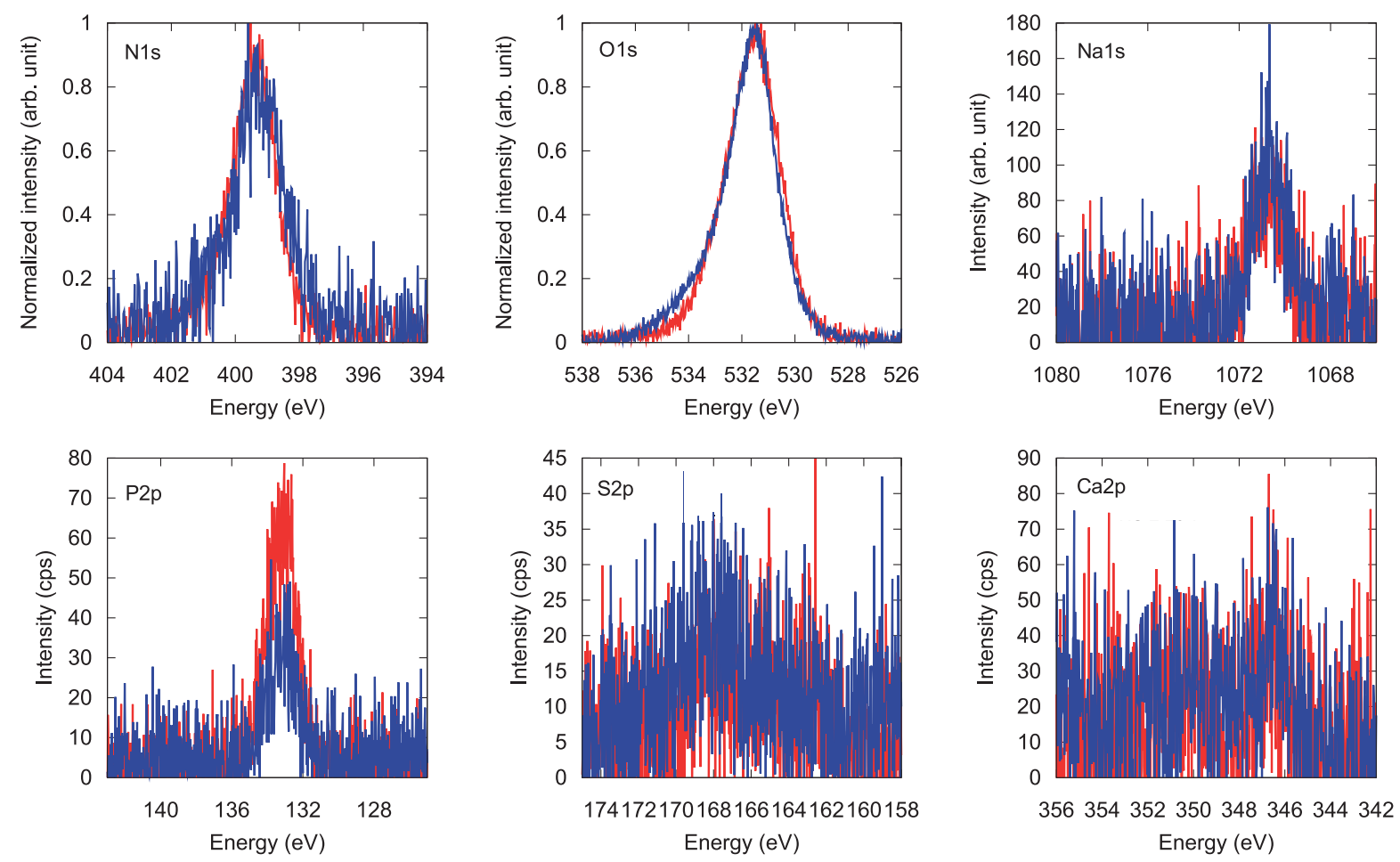

Native dragline

Washed dragline

FIGURE 5 |XPS spectra for different elements in native spider dragline (red) and spider dragline after an ether wash (blue).

TABLE 1 |XPS characterization of different elements and components of native spider dragline and spider dragline after an ether wash.

\begin{tabular}{|c|c|c|c|c|c|c|c|c|c|}
\hline$\%$ (Standard deviation) & C 1s C-H & C 1s $C=0$ & C 1s C-O & N 1s & $01 \mathrm{~s}$ & $\mathrm{Na} 1 \mathrm{~s}$ & P 2p & S 2p & $\mathrm{Ca} 2 p$ \\
\hline Native dragline & $60.1(6.3)$ & $6.8(2.3)$ & $16.0(2.5)$ & $2.8(1.4)$ & $8.8(2.7)$ & $1.2(0.7)$ & $0.68(0.06)$ & $0.53(0.16)$ & $0.75(0.45)$ \\
\hline Washed dragline & $50.1(2.6)$ & $8.2(2.1)$ & $16.3(3.2)$ & $2.4(0.1)$ & $13.5(3.7)$ & $1.4(0.5)$ & $0.48(0.25)$ & $0.74(0.08)$ & $1.0(0.53)$ \\
\hline
\end{tabular}

Numbers in parentheses are standard deviations in results obtained three different measurement positions. 
unexpected contamination from the washing experiment. We could not detect potassium in the wide-range XPS spectra, even though the presence of potassium is expected according to the previous ionic elemental analyses of the major ampullate glands (Knight and Vollrath, 2001; Andersson et al., 2014; Oktaviani et al., 2019). Perhaps, potassium might not be localized at the surface of the dragline. On the other hand, O 1s, C 1s, and $\mathrm{N} 1 \mathrm{~s}$ were obviously detected as shown in Figures 4, 5 .

The spectra concerning $\mathrm{C} 1 \mathrm{~s}$ were further assigned as shown in Figure 4, namely, $\mathrm{C}=\mathrm{O}, \mathrm{C}-\mathrm{O} / \mathrm{C}-\mathrm{N}$, and $\mathrm{C}-\mathrm{C} / \mathrm{C}-\mathrm{H}$. The XPS spectra were deconvoluted with the Gaussian-Lorentzian mixed function to resolve multiple chemical states, as listed in Table 1. The standard deviations of the contents include all the errors from the variation of the atomic concentration of the samples, positional accuracy of the fitting, and also from the equipment itself. We think the primary origin of the deviation might be the variation of the samples themselves because our samples are natural ones. The increase in $\mathrm{C}=\mathrm{O}$ content suggests the appearance of peptide bonds at the surface after the washing process. The removal of the lipid layer via the diethyl ether treatment was successfully detected by XPS. Thus, the XPS measurements with spider draglines indicated sufficient quantification to discuss the surface chemical compositions.

Figure 5 shows each spectrum corresponding to $\mathrm{N} 1 \mathrm{~s}, \mathrm{O} 1 \mathrm{~s}$, $\mathrm{Na} 1 \mathrm{~s}, \mathrm{P} 2 \mathrm{p}, \mathrm{S} 2 \mathrm{p}$, and Ca 2p. Only phosphate, P 2p, decreased in intensity after the skin layer removal. Considering that phosphate ions exist in the overall ampullate gland of spiders (Andersson et al., 2014; Oktaviani et al., 2019), the skin layer may contain more phosphate ions than the protein core inside the dragline. The peak corresponding to calcium, Ca $2 \mathrm{p}$, could be background noise because of the abundant presence of calcium ions in the natural environment. The other elements, $\mathrm{N} 1 \mathrm{~s}, \mathrm{O} 1 \mathrm{~s}, \mathrm{Na} 1 \mathrm{~s}$, and $S 2 p$, did not show any significant change before and after the skin removal treatment. Sodium should be related to the ionic conditions of the spider ampullate gland, but we could not find any related difference between the skin layer and core regions. The $S 2 p$ peak, which can be related to sulfate ions and the cysteines of spider silk proteins, was detected even though its intensity and signal-to-noise ratio were very low. The sulfate ion is kosmotropic and can be present at the end of the spider major ampullate gland to form the dragline; hence, it is reasonable to detect the $S 2 p$ peak by XPS. However, we gave up discussing more details of the $S 2$ p peak, due to the relatively low intensity. The $\mathrm{Ca} 2 \mathrm{p}$ peak can be related to $\mathrm{Ca}$ ions, which are predominantly present under the natural environments. Similar

\section{REFERENCES}

Andersson, M., Chen, G., Otikovs, M., Landreh, M., Nordling, K., Kronqvist, N., et al. (2014). Carbonic anhydrase generates $\mathrm{CO} 2$ and $\mathrm{H}+$ that drive spider silk formation via opposite effects on the terminal domains. PLoS Biol. 12:e1001921. doi: 10.1371/journal.pbio.1001921

Augsten, K., Muhlig, P., and Herrmann, C. (2000). Glycoproteins and skincore structure in Nephila clavipes spider silk observed by light and electron microscopy. Scanning 22, 12-15. doi: 10.1002/sca.4950220103 to the S 2p peak, we avoid to discuss the more details of the Ca $2 p$ based on the current signal-to-noise ratios.

\section{CONCLUSION}

We evaluated the surface of Nephila clavata spider dragline by FE-SEM and XPS to obtain clear surface morphological and chemical information. This is the first surface analysis of natural spider draglines by both powerful techniques. The FE-SEM images of the spider dragline indicate that the spider dragline forms a bundle of microfibrils. This hierarchical structure might induce faint fibril and network-like patterns at the surface of the dragline, however, we need to further study the origin of the patterns. Perhaps, we need to consider the effect of the natural spinning process with spider spinnerets to explain the surface patterns found in this study. XPS analysis revealed the presence of $\mathrm{Na}, \mathrm{P}$, and $\mathrm{S}$, which are reasonably explained by considering the biological components of the major ampullate gland of spiders. The information obtained here is preliminary but will be important and essential to consider the molecular transition of silk proteins to form excellent hierarchical structures during the spider dragline spinning process.

\section{DATA AVAILABILITY STATEMENT}

The raw data supporting the conclusions of this article will be made available by the authors, without undue reservation, to any qualified researcher.

\section{AUTHOR CONTRIBUTIONS}

HS prepared the samples. AT conducted the FE-SEM measurements. KNa performed the XPS experiments. KT and $\mathrm{KNu}$ conceptualized and oversaw the project. All the authors co-wrote the manuscript.

\section{FUNDING}

This work was financially supported by the Impulsing Paradigm Change through Disruptive Technologies Program (ImPACT) of the Japan Science and Technology Corporation (JST) and RIKEN Engineering Network.

Blackledge, T. A. (2012). Spider silk: a brief review and prospectus on research linking biomechanics and ecology in draglines and orb webs. J. Arachnol. 40, 1-12. doi: 10.1636/M11-67.1

Brown, C. P., Harnagea, C., Gill, H. S., Price, A. J., Traversa, E., Licoccia, S., et al. (2012). Rough fibrils provide a toughening mechanism in biological fibers. ACS Nano 6, 1961-1969. doi: 10.1021/nn300130q

Dao, A. T. N., Nakayama, K., Shimokata, J., and Taniike, T. (2017). Multilateral characterization of recombinant spider silk in thermal degradation. Polym. Chem. 8, 1049-1060. doi: 10.1039/c6py01954d 
Faugas, B., Ellison, M. S., Dean, D., and Kennedy, M. S. (2013). Surface characterization of as-spun and supercontracted Nephila clavipes dragline silk. J. Surf. Eng. Mater. Adv. Technol. 03, 18-26. doi: 10.4236/jsemat.2013.33A004

Frische, S., Maunsbach, A. B., and Vollrath, F. (1998). Elongate cavities and skin-core structure in Nephila spider silk observed by electron microscopy. J. Microsc. 189, 64-70. doi: 10.1046/j.1365-2818.1998.00285.x

Fu, C. J., Porter, D., and Shao, Z. Z. (2009). Moisture effects on Antheraea pernyi silk's mechanical property. Macromolecules 42, 7877-7880. doi: 10.1021/ Ma901321k

Gosline, J. M., DeMont, M. E., and Denny, M. W. (1986). The structure and properties of spider silk. Endeavour 10, 37-43. doi: 10.1016/0160-9327(86) 90049-9

Heim, M., Romer, L., and Scheibel, T. (2010). Hierarchical structures made of proteins. The complex architecture of spider webs and their constituent silk proteins. Chem. Soc. Rev. 39, 156-164. doi: 10.1039/b813273a

Holland, C., Numata, K., Rnjak-Kovacina, J., and Seib, F. P. (2018). The biomedical use of silk: past, present, future. Adv. Healthc. Mater. 8:e1800465. doi: 10.1002/ adhm.201800465

Knight, D. P., and Vollrath, F. (2001). Changes in element composition along the spinning duct in a Nephila spider. Naturwissenschaften $88,179-182$. doi: $10.1007 / \mathrm{s} 001140100220$

Li, M., Ogiso, M., and Minoura, N. (2003). Enzymatic degradation behavior of porous silk fibroin sheets. Biomaterials 24, 357-365. doi: 10.1016/s01429612(02)00326-5

Li, S. F., McGhie, A. J., and Tang, S. L. (1994). New internal structure of spider dragline silk revealed by atomic force microscopy. Biophys. J. 66, 1209-1212. doi: 10.1016/s0006-3495(94)80903-8

Li, S. F. Y., McGhie, A. J., and Tang, S. L. (1994). Comparative study of the internal structures of Kevlar and spider silk by atomic force microscopy. J. Vac. Sci. Technol. A 12, 1891-1894. doi: 10.1116/1.578978

Lin, T. Y., Masunaga, H., Sato, R., Malay, A. D., Toyooka, K., Hikima, T., et al. (2017). Liquid crystalline granules align in a hierarchical structure to produce spider dragline microfibrils. Biomacromolecules 18, 1350-1355. doi: 10.1021/ acs.biomac.7b00086

Malay, A. D., Arakawa, K., and Numata, K. (2017). Analysis of repetitive amino acid motifs reveals the essential features of spider dragline silk proteins. PLoS One 12:e0183397. doi: 10.1371/journal.pone.0183397

Malay, A. D., Sato, R., Yazawa, K., Watanabe, H., Ifuku, N., Masunaga, H., et al. (2016). Relationships between physical properties and sequence in silkworm silks. Sci. Rep. 6:27573. doi: 10.1038/srep27573

Numata, K., Cebe, P., and Kaplan, D. L. (2010). Mechanism of enzymatic degradation of beta-sheet crystals. Biomaterials 31, 2926-2933. doi: 10.1016/J. Biomaterials.2009.12.026

Numata, K., Finne-Wistrand, A., Albertsson, A. C., Doi, Y., and Abe, H. (2008). Enzymatic degradation of monolayer for poly(lactide) revealed by real-time atomic force microscopy: effects of stereochemical structure, molecular weight, and molecular branches on hydrolysis rates. Biomacromolecules 9, 2180-2185. doi: 10.1021/Bm800281d

Numata, K., and Kaplan, D. L. (2010). Mechanisms of enzymatic degradation of amyloid beta microfibrils generating nanofilaments and nanospheres related to cytotoxicity. Biochemistry 49, 3254-3260. doi: 10.1021/bi902134p

Numata, K., Kikkawa, Y., Tsuge, T., Iwata, T., Doi, Y., and Abe, H. (2005). Enzymatic degradation processes of poly[(R)-3-hydroxybutyric acid] and
poly[(R)-3-hydroxybutyric acid-co-(R)-3-hydroxyvaleric acid] single crystals revealed by atomic force microscopy: effects of molecular weight and second-monomer composition on erosion rates. Biomacromolecules 6, 20082016. doi: 10.1021/bm0501151

Numata, K., Sato, R., Yazawa, K., Hikima, T., and Masunaga, H. (2015). Crystal structure and physical properties of Antheraea yamamai silk fibers: long poly(alanine) sequences are partially in the crystalline region. Polymer 77, 87-94. doi: 10.1016/j.polymer.2015.09.025

Oktaviani, N. A., Matsugami, A., Hayashi, F., and Numata, K. (2019). Ion effects on the conformation and dynamics of repetitive domains of a spider silk protein: implications for solubility and beta-sheet formation. Chem. Commun. 55, 9761-9764. doi: 10.1039/c9cc03538a

Riekel, C., Burghammer, M., Dane, T. G., Ferrero, C., and Rosenthal, M. (2017). Nanoscale structural features in major ampullate spider silk. Biomacromolecules 18, 231-241. doi: 10.1021/acs.biomac.6b01537

Rousseau, M.-E., Hernández Cruz, D., West, M. M., Hitchcock, A. P., and Pézolet, M. (2007). Nephila clavipes spider dragline silk microstructure studied by scanning transmission X-ray microscopy. J. Am. Chem. Soc. 129, 3897-3905. doi: $10.1021 / \mathrm{ja067471 \textrm {r }}$

Shao, Z., Hu, X. W., Frische, S., and Vollrath, F. (1999). Heterogeneous morphology of Nephila edulis spider silk and its significance for mechanical properties. Polymer 40, 4709-4711. doi: 10.1016/s0032-3861(99) 00072-5

Sponner, A., Vater, W., Monajembashi, S., Unger, E., Grosse, F., and Weisshart, K. (2007). Composition and hierarchical organisation of a spider silk. PLoS One 2:e998. doi: 10.1371/journal.pone.0000998

Thiel, B. L., Kunkel, D. D., and Viney, C. (1994). Physical and chemical microstructure of spider dragline: a study by analytical transmission electron microscopy. Biopolymers 34, 1089-1097. doi: 10.1002/bip.36034 0812

Vollrath, F. (1992). Spider webs and silks. Sci. Am. 266, 70-77.

Vollrath, F., Holtet, T., Thogersen, H. C., and Frische, S. (1996). Structural organization of spider silk. Proc. R. Soc. Lond. B 263, 147-151. doi: 10.1098/ rspb.1996.0023

Yazawa, K., Ishida, K., Masunaga, H., Hikima, T., and Numata, K. (2016). Influence of water content on the beta-sheet formation, thermal stability, water removal, and mechanical properties of silk materials. Biomacromolecules 17, 1057-1066. doi: 10.1021/acs.biomac.5b01685

Yazawa, K., Malay, A. D., Masunaga, H., and Numata, K. (2019). Role of skin layers on mechanical properties and supercontraction of spider dragline silk fiber. Macromol. Biosci. 19:e1800220. doi: 10.1002/mabi.201800220

Conflict of Interest: The authors declare that the research was conducted in the absence of any commercial or financial relationships that could be construed as a potential conflict of interest.

Copyright (C) 2020 Sogawa, Nakano, Tateishi, Tajima and Numata. This is an openaccess article distributed under the terms of the Creative Commons Attribution License (CC BY). The use, distribution or reproduction in other forums is permitted, provided the original author(s) and the copyright owner(s) are credited and that the original publication in this journal is cited, in accordance with accepted academic practice. No use, distribution or reproduction is permitted which does not comply with these terms. 